\title{
Caninos incluidos, tratamiento odontológico. Revisión de la literatura
}

\section{Unerupted canines, dental treatment. A literature review}

\author{
Egido Moreno S*, Arnau Matas C*, Juárez Escalona I**, Jané-Salas E***, \\ Marí Roig A****, López-López J***
}

\section{RESUMEN}

El objetivo de este trabajo es realizar una revisión bibliográfica de los últimos 5 años (2006-2011), sobre la situación actual de los caninos incluidos y su tratamiento. Se ha analizado la incidencia, etiología, diagnóstico y factores pronósticos de su alineamiento, así como las terapéuticas de los mismos. Su manejo es de especial importancia, ya que estos dientes tienen un papel fundamental en la apariencia facial, estética dental, desarrollo del arco dental y la oclusión funcional.

Se deben diagnosticar mediante una evaluación clínica y radiológica minuciosa además de un examen radiográfico; determinando las posibles complicaciones asociadas y las opciones de tratamiento individualizándolas en cada caso. Se han planteado diferentes formas de manejarlos que van desde los controles periódicos, la prevención de la inclusión con el tratamiento interceptivo, el tratamiento ortodóncico-quirúrgico o la extracción. Antes de iniciar cualquier procedimiento debemos valorar las características individuales de cada paciente, así como la situación y la inclinación del diente para lograr nuestro objetivo.

Palabras clave: Canino, incluido, tratamiento, ortodóncico y quirúrgico.

\section{SUMMARY}

The aim of this article is to make a bibliographical review, from studies performed in the last 5 years (20062011), to analyse the current trends of the unerupted canines in its epidemiology, aetiology, diagnostic methods, prognostic factors in their alignment; and the various treatment options available. It is especially important because of its relevance in the facial appearance, dental aesthetics and functional occlusion.

The diagnosis of the unerupted canine should be made through a thorough clinical and radiographic examination, because it helps in determining the best treatment plan and prevention and/or evaluation of possible complications. Different ways to manage such cases have been suggested from periodic controls, preventing the impaction using interceptive treatment, surgical and orthodontic techniques, and lastly, extraction. Thus, before commencing with any treatment, a proper evaluation of the patient characteristics and the characteristics of the canine should be evaluated to achieve the best possible outcome.

Key words: Canine, impacted, treatment, orthodontic and surgical.

* Alumna del máster de Medicina, Cirugía e Implantología. Facultad de Odontología. Universidad de Barcelona.

** Profesor del máster Medicina, Cirugía e Implantología. Facultad de Odontología. Universidad de Barcelona. Médico especialista en Cirugía Maxilofacial. Servicio de Cirugía Maxilofacial. Hospital Universitario de Bellvitge.

*** Profesor del máster Medicina, Cirugía e Implantología. Facultad de Odontología. Universidad de Barcelona. Profesor del Medicina Oral. Facultad de Odontología. Universidad de Barcelona.

**** Profesor del máster Medicina, Cirugía e Implantología. Facultad de Odontología. Universidad de Barcelona. Médico especialista en Cirugía Maxilofacial. Servicio de Cirugía Maxilofacial. Responsable de la Unidad Funcional de Cabeza y Cuello del Hospital Universitario de Bellvitge. 
Fecha de recepción: 1 de noviembre de 2012.

Aceptado para publicación: 20 de noviembre de 2012.

Egido Moreno S, Arnau Matas C, Juárez Escalona I, Jané-Salas E, Marí Roig A, López-López J. Caninos incluidos, tratamiento odontológico. Revisión de la literatura. Av. Odontoestomatol 2013; 29 (5): 227-238.

\section{INTRODUCCIÓN}

Los caninos maxilares y mandibulares juegan un papel importante en la apariencia facial, estética dental, desarrollo del arco dental y la oclusión funcional (1). El canino maxilar tiene el periodo de desarrollo más largo, el área más profunda de desarrollo y un camino más difícil para llegar a su posición oclusal $(2,3)$. Por esta razón la inclusión del canino es la más frecuente, sobre todo el maxilar, con una incidencia del 0,2 al 3,6\% (3-5).

Para colocar el diente en su posición y así evitar complicaciones derivadas de esta inclusión se han propuesto diferentes técnicas. Aunque sólo con un tratamiento multidisciplinar que incluya ortodoncistas, periodoncistas y cirujanos orales, el tratamiento será satisfactorio $(1,6)$.

En este artículo se revisan las causas y el diagnostico de la retención de los caninos. También se presentan las diferentes técnicas para exponerlos. Estos aspectos son de especial importancia para los clínicos por el papel estético y funcional de los caninos y por su alta frecuencia de inclusión (1). Actualmente no se conocen exactamente las causas de la falta de erupción y consecuentemente no hay pautas claras de actuación atendiendo a la prevención así como al subsiguiente tratamiento (7).

\section{MATERIALES Y MÉTODOS}

Se realizó una revisión bibliográfica de los últimos 5 años (2006-2011), excepto 1 artículo de 2004 por la información que nos aporta sobre la etiología de estas agenesias, en las bases de datos Medline/Pubmed, Cochrane y Scielo.

Con las palabras clave: canine, impacted, treatment, orthodontic and surgical; se encontraron 53 artícu- los, de los cuáles se escogieron 33 para la revisión, ya que se eliminaron aquellos con bajo nivel de evidencia (opiniones de expertos o artículos a propósito de un caso). También se descartaron los que hacían referencia a caninos transmigrados, exceptuando dos de ellos por su implicación en el tema del artículo, ya que aportaban información epidemiológica y etiológica relevante. Los artículos seleccionados fueron en inglés y castellano.

\section{RESULTADOS}

Después de la selección de estos 32 artículos (Figura 1), la información obtenida se ha clasificado en 5 apartados (epidemiología, etiología, diagnóstico, factores pronósticos en el alineamiento y opciones de tratamiento de los caninos incluidos) y, de esta forma, obtener una visión más clara de la situación actual para afrontar los caninos incluidos. En base a estos apartados revisaremos el tema en la discusión.

\section{DISCUSIÓN}

\section{INCIDENCIA}

Después de los terceros molares el canino maxilar es el segundo diente en frecuencia de retención $(1,6,8,9)$, con una incidencia del $0,2 \%$ al 3,6\% según los autores (1,6,7-18). En relación a su posición, comúnmente los encontramos en palatino $(1,7,8)$, en una proporción del $61 \%$ y en una posición vestibular en el 5\%, mientras que el $34 \%$ restante se encuentra en línea con el arco dental (7). Los caninos incluidos tienen el doble de incidencia en el sexo femenino $(8,9)$ y en un $8-10 \%$ de los casos la inclusión es bilateral (8). La incidencia de caninos incluidos en la mandíbula es mucho menor, entre un 0,35$1,29 \%(2,8)$. 


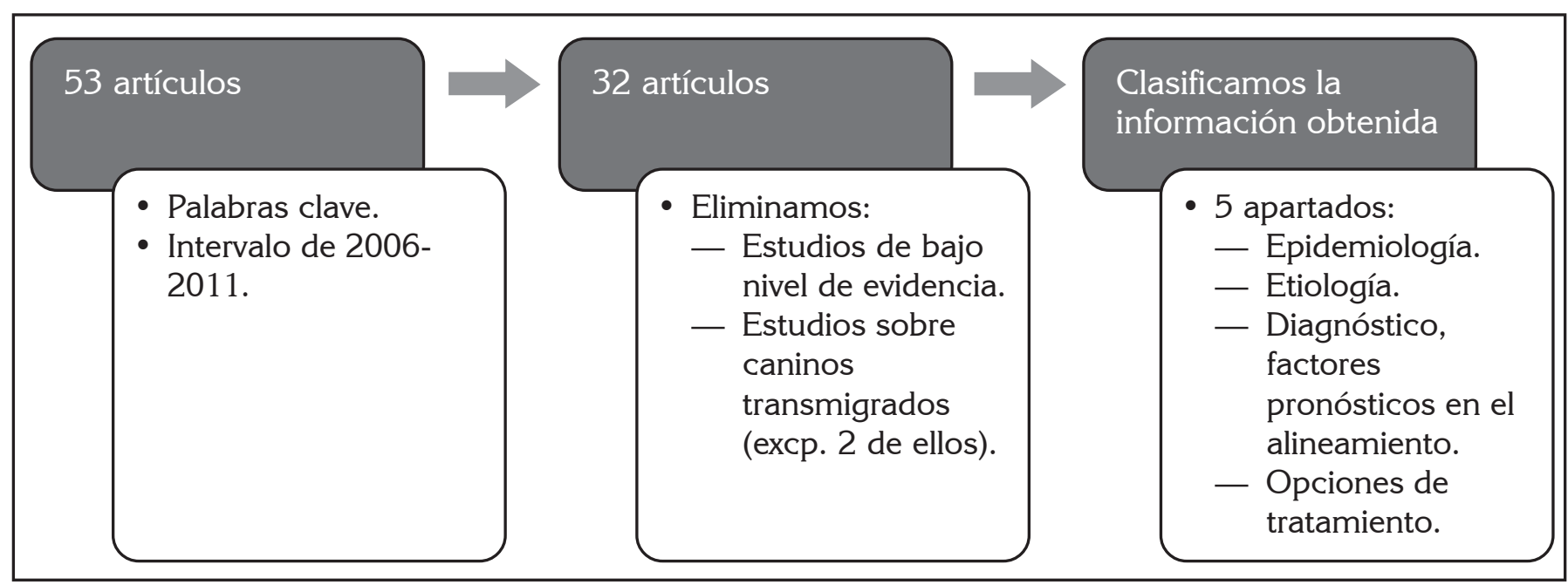

Fig. 1. Método de selección de los artículos revisados.

\section{ETIOLOGÍA}

La etiología de los caninos incluidos es desconocida (7). Aunque se han propuesto diferentes causas que pueden ser locales, generales o genéticas. Las causas generales abarcan enfermedades sistémicas como cuadros febriles, irradiación $(1,8)$, síndrome de Gardner, disostosis cleidocraneal $(10,11,19)$ o deficiencias endocrinas $(2,20)$. Entre las causas locales están las discrepancias óseo-dentarias, pérdida temprana o retención prolongada del canino temporal, anquilosis, quistes o formaciones neoplásicas, dientes supernumerarios, dilaceraciones de la raíz, cierre prematuro del ápice, iatrogenia, trauma, etc. $(2,6,12,19,20)$. Dentro de estas causas locales, en el caso de los caninos incluidos maxilares desplazados hacia palatino encontramos la teoría de la orientación que sugiere que el canino erupciona gracias a la guía que le proporciona el incisivo lateral, si éste está ausente o malformado el canino no erupciona correctamente $(1,6,7,21)$.

Finalmente, también se ha descrito la teoría genéti$\mathrm{ca}$, que hace referencia a factores genéticos como principal origen $(1,6,7,21)$; como, por ejemplo, la posición anormal del germen dental, factores hereditarios y el paladar hendido (1,9-11). Esta teoría es defendida por algunos autores, como Kolenc-Fusé que revisa las alteraciones genéticas que pueden ser responsables de las agenesias dentarias. Estas mutaciones de han encontrado, sobretodo, ligadas a los factores de transcripción MSX1 y PAX9 (22).

\section{COMPLICACIONES}

Dada la importancia del canino, su no tratamiento podría comportar un compromiso estético, fonético y masticatorio (19). Otras complicaciones de no tratar los caninos incluidos pueden resultar en el desplazamiento o pérdida de vitalidad de los incisivos, acortamiento del arco dental, formación de quistes foliculares, anquilosis del canino, infecciones y dolores recurrentes, reabsorción interna, reabsorción externa del diente adyacente o combinaciones de todos estos factores $(6,7,13)$. La reabsorción externa del diente adyacente es la mayor preocupación y la complicación más común que puede provocar incluso la pérdida del diente $(2,6,7,13)$. Por otra parte, la presencia de caninos incluidos puede no causar efectos perjudiciales durante toda la vida del paciente. Aunque el potencial riesgo de complicaciones recalcan la necesidad de tratamiento o monitorización de éstos (1).

\section{DIAGNÓSTICO}

Los diferentes métodos diagnósticos, evaluación clínica y radiográfica, para la detección temprana y prevención de los caninos incluidos pueden reducir el tiempo de tratamiento, la complejidad, complicaciones y el coste (7). La evaluación clínica incluye la historia familiar, la inspección visual (7) y la palpación de la protuberancia sobre el canino primario. Posibles signos clínicos de inclusión son: persisten- 
cia del canino deciduo más allá de los 14 o 15 años, asimetría en la protuberancia canina, presencia de una protuberancia en palatino, erupción dental retrasada o migración del incisivo lateral (8).

Se ha descrito que el $29 \%$ de los caninos no son palpables a los 10 años, el 5\% a los 11 años y el 3\% posteriormente. Por esta razón es esencial el examen radiológico y se debe combinar con la evaluación clínica (8). Se pueden usar técnicas en dos dimensiones -2D - como radiografías periapicales, oclusales y panorámicas $(15,22)$. También podemos usar combinaciones de éstas para determinar su posición en el arco dental, como por ejemplo la técnica de Clark $(1,8)$. Estas técnicas en 2D, sobretodo la radiografía panorámica, nos proporcionan información útil sobre la edad dental, simetría, número de dientes presentes, secuencia de la erupción dental, y la presencia de patología o variaciones respecto a la norma. A pesar de esto, las técnicas en 2D tienen la desventaja de la distorsión y superposición de estructuras que pueden reducir la precisión de diagnóstico. Además, las técnicas de imagen convencionales radiográficas se han descrito como inadecuadas para el diagnóstico de la reabsorción radicular, una complicación frecuentemente asociada $(7,23)$.

Para poder observar con detalle la reabsorción de raíces o la localización exacta del canino podemos usar la tomografía computarizada (TC) que nos proporcionará información en tres dimensiones - 3D(19). El mayor inconveniente es la alta dosis de radiación que es particularmente nociva para los niños ya que son más sensibles a la radiación que los adultos (24). Por este motivo se han introducido de manera muy importante en el campo de la odontología, los Cone-beam TC (CBTC) que reducen la exposición a la radiación. Con estos dispositivos se puede reconstruir en 3D el área de interés. Esto nos proporcionará información para elaborar un correcto diagnóstico de la posición sin superposiciones en los planos sagital, axial y coronal $(1,7,19)$.

\section{FACTORES PRONÓSTICOS EN EL}

\section{ALINEAMIENTO DEL CANINO INCLUIDO}

El alineamiento en la arcada dental de estos dientes puede requerir tratamientos complicados y prolon- gados, por lo que su extracción se debe considerar si es poco probable la alineación final del diente en el arco dental. Para determinar el tipo de tratamiento a efectuar se deberán considerar diferentes factores que influirán en el pronóstico del tratamiento:

- Cooperación del paciente: No acudir a las citas o una pobre higiene oral puede afectar al resultado del tratamiento (25).

- Edad del paciente: Cuanto mayor sea la edad el paciente más duración y menor éxito tendrá el tratamiento (26).

- Presencia de espacio o apiñamiento: En el 85\% de los casos en que hay suficiente espacio en la arcada dental el canino se encuentra en palatino, mientras que en los casos de apiñamiento es más común encontrarlo en posición vestibular.

- Posición del canino: Para determinar un pronóstico nos basaremos más en la posición inicial mesiodistal y vertical que en la inclinación del diente incluido (11). De esta manera, se prevé un buen pronóstico cuando el extremo del borde incisal del canino está a nivel de la unión amelocementaria del incisivo adyacente. Un pronóstico aceptable cuando el extremo del borde incisal se encuentra en la mitad de la longitud de la raíz del diente contiguo. Finalmente, se predice un diagnóstico pobre cuando el extremo del borde incisal del canino está en el tercio apical de la raíz del incisivo (25).

\section{TRATAMIENTO DE LOS CANINOS INCLUIDOS}

Son variadas las posturas terapéuticas propuestas en los diferentes trabajos revisados.

\section{Control}

Se puede optar por no tratar el canino incluido. El canino primario puede tener una buena longitud de raíz y puede ser estéticamente aceptable. Por otra parte, el canino deciduo se puede haber exfoliado más temprano y el espacio del canino haberse cerrado espontáneamente creando un buen punto de contacto incisivo-premolar (2). También se podría optar por no tratar el canino si éste está incluido muy profundo sin ninguna patología asociada, particularmente en pacientes mayores (27). Si optamos 
por la abstención terapéutica, debemos realizar controles clínicos cada 6-12 meses y radiográficos cada 2-3 años del canino definitivo para asegurarnos que no se produce ninguna complicación (2).

\section{Tratamiento interceptivo}

Se basa en la extracción del canino temporal para prevenir la impactación del canino definitivo. Esta técnica se fundamenta en la teoría que la persistencia del canino primario puede representar un obstáculo para la emergencia del diente definitivo (6). Este tipo de tratamiento se debe efectuar en niños con suficiente espacio en la arcada dental (mayores de 11 años (11) y menores de 13 años) (6). El canino definitivo debería emerger en los 12 meses siguientes, si no es así no se espera que éste pueda erupcionar y se deberá evaluar otro tratamiento (6). Este tratamiento estaría sobretodo indicado en aquellos casos en que la corona del canino está distal de la raíz del incisivo lateral $(1,6)$ y las posibilidades de ubicar el canino a su posición habitual en la arcada serán inversamente proporcionales a medida que aumenta la inclinación horizontal (1).

Alessandri-Bonetti et al. (14) en su estudio de casos controles de 60 niños entre 9 y 12 años, propuso como tratamiento interceptivo la exodoncia del canino y del primer molar temporales. Según el estudio se logró un mayor porcentaje de éxito además de incrementar el paralelismo entre las raíces del canino permanente y del incisivo adyacente, lo que reduce el riesgo de reabsorción de la raíz del incisivo lateral (14).

\section{Tratamiento ortodóncico-quirúrgico}

El mejor abordaje es el diagnóstico temprano y la intercepción de un potencial canino incluido. En ausencia de esta prevención, se debe considerar el tratamiento ortodóncico-quirúrgico para exponer el canino y llevarlo a oclusión (28) (Tablas 1 y 2).

\section{TABLA 1.- TÉCNICAS QUIRÚRGICAS PARA LA EXPOSICIÓN DE LOS CANINOS INCLUIDOS MAXILARES EN POSICIÓN VESTIBULAR $(1,7)$}

\begin{tabular}{|l|l|l|l|l|}
\hline Técnica & \multicolumn{1}{|c|}{ Indicación } & \multicolumn{1}{|c|}{$\begin{array}{l}\text { Iniciación } \\
\text { de la tracción } \\
\text { ortodóncica }\end{array}$} & Ventajas & Inconvenientes \\
\hline Gingivectomía & $\begin{array}{l}\text { El extremo de la cúspide se } \\
\text { localiza coronal a la unión } \\
\text { mucogingival, con una adecuada } \\
\text { cantidad de encía queratinizada, el } \\
\text { diente no está cubierto por hueso. }\end{array}$ & $\begin{array}{l}\text { Normalmente no } \\
\text { es necesaria la } \\
\text { tracción } \\
\text { ortodóncica. }\end{array}$ & $\begin{array}{l}\text { Fácil de } \\
\text { realizar. }\end{array}$ & $\begin{array}{l}\text { Pérdida de encía } \\
\text { queratinizada, } \\
\text { posible daño } \\
\text { periodontal, posible } \\
\text { sobrecrecimiento } \\
\text { gingival en la zona } \\
\text { de la cirugía. }\end{array}$ \\
\hline $\begin{array}{l}\text { Colgajo de } \\
\text { reposición } \\
\text { apical }\end{array}$ & $\begin{array}{l}\text { La corona del canino está apical a } \\
\text { la línea mucogingival, encía } \\
\text { adherida <3 mm. }\end{array}$ & $\begin{array}{l}\text { Dos o tres } \\
\text { semanas después } \\
\text { de la cirugía. }\end{array}$ & $\begin{array}{l}\text { Conservación } \\
\text { de la encía } \\
\text { queratinizada. }\end{array}$ & $\begin{array}{l}\text { Aumenta el riesgo } \\
\text { de recesión gingival, } \\
\text { más traumática. }\end{array}$ \\
\hline Técnica cerrada & $\begin{array}{l}\text { El diente está en el centro del } \\
\text { alveolo, la corona está apical a la } \\
\text { línea mucogingival. }\end{array}$ & $\begin{array}{l}\text { Una o dos } \\
\text { semanas después } \\
\text { de la cirugía. }\end{array}$ & $\begin{array}{l}\text { Mayor } \\
\text { estética, } \\
\text { facilita el } \\
\text { movimiento } \\
\text { del diente. }\end{array}$ & $\begin{array}{l}\text { En caso de } \\
\text { despegamiento se } \\
\text { debe realizar una } \\
\text { segunda cirugía, } \\
\text { posibles problemas } \\
\text { mucogingivales. }\end{array}$ \\
\hline
\end{tabular}




\section{TABLA 2.- TÉCNICAS QUIRÚRGICAS PARA LA EXPOSICIÓN DE LOS CANINOS INCLUIDOS MAXILARES EN POSICIÓN PALATINA}

\begin{tabular}{|c|c|c|c|c|}
\hline Técnica & Indicación & $\begin{array}{l}\text { Iniciación } \\
\text { de la tracción } \\
\text { ortodóncica }\end{array}$ & Ventajas & Inconvenientes \\
\hline Técnica cerrada & $\begin{array}{l}\text { El canino se localiza cercano } \\
\text { al incisivo central y lateral, } \\
\text { horizontal y superior al techo } \\
\text { de la boca }(1,7) \text {. }\end{array}$ & $\begin{array}{l}\text { Una o dos } \\
\text { semanas después } \\
\text { de la cirugía }(1,7) \text {. }\end{array}$ & $\begin{array}{l}\text { Inmediata tracción } \\
\text { ortodóncica( }(1,7) \text {. }\end{array}$ & $\begin{array}{l}\text { Necrosis ósea, } \\
\text { reabsorción } \\
\text { radicular, fallos en } \\
\text { la adhesión }(1,7) \text {. }\end{array}$ \\
\hline Técnica abierta & $\begin{array}{l}\text { El canino se localiza cercano } \\
\text { al incisivo central y lateral, } \\
\text { horizontal y superior al techo } \\
\text { de la boca }(1,7) \text {. }\end{array}$ & $\begin{array}{l}\text { Una o dos } \\
\text { semanas después } \\
\text { de la cirugía }(1,7) \text {. }\end{array}$ & $\begin{array}{l}\text { Mejor visualización } \\
\text { de la corona y } \\
\text { mejor dirección del } \\
\text { movimiento del } \\
\text { diente }(1,7) \text {. }\end{array}$ & $\begin{array}{l}\text { Sobrecrecimiento } \\
\text { gingival }(1,7) \text {. }\end{array}$ \\
\hline Tunelización & $\begin{array}{l}\text { Presencia del canino } \\
\text { primario }(1,4) .\end{array}$ & $\begin{array}{l}\text { Después de la } \\
\text { retirada de la } \\
\text { sutura }(10 \text { días } \\
\text { aprox.) }(1,7) .\end{array}$ & $\begin{array}{l}\text { El canino } \\
\text { permanente es } \\
\text { guiado hacia el } \\
\text { alveolo del canino } \\
\text { primario }(1,4) .\end{array}$ & $\begin{array}{l}\text { Requiere la } \\
\text { presencia del } \\
\text { canino primario } \\
(1,4) .\end{array}$ \\
\hline
\end{tabular}

\section{Tratamiento ortodóncico prequirúrgico}

Antes de cualquier tratamiento, se debe crear espacio suficiente para poder posicionarlo, ya que en caso contrario perderíamos longitud de arcada por la mesialización del diente posterior y la distalización del anterior. Además al poner brackets en todo el arco dental se obtiene el suficiente anclaje para poder traccionarlo. Otra opción es colocar un microimplante o miniimplante como anclaje que al acabar el tratamiento se puede quitar fácilmente (8).

\section{2a. Técnicas para exponer el canino maxilar incluido}

Ya hemos comentado que lo mas común es que se presente por palatino, en ese caso se debería optar por exponer el diente y permitir que erupcione naturalmente durante la dentición mixta, o bien adhiriendo un accesorio al diente y usar fuerzas ortodóncicas para moverlo (1).

Disponemos de diferentes técnicas para exponer el canino (Tabla 3): Gingivectomía, colgajo de reposi- ción apical, técnica cerrada, técnica abierta (Figura 2) y tunelización. En cualquier caso, para usar las diferentes técnicas se ha de tener en cuenta criterios como: la posición labio-lingual del canino incluido, la posición vertical del diente en relación a la unión mucogingival, la cantidad de encía queratinizada y la posición mesiodistal de la corona del canino (1) (Figura 3).

Para determinar la técnica quirúrgica a usar, también se debe tener en cuenta la prevención de posibles defectos periodontales después del tratamiento en esta zona. Hay estudios que muestran que usando una técnica cerrada se obtiene un menor defecto periodontal. Estos estudios son retrospectivos y no se analiza la gravedad de impactación de los caninos. Otros muestran que los resultados no varían de una a otra técnica $(13,29)$, aunque la capacidad de recuperación si es menor en la técnica abierta, puede que por este motivo se considere que el resultado final es peor con esta técnica (20).

Así que para decidir el tipo de tratamiento a seguir para obtener el mejor resultado final, se deberá considerar la profundidad del canino incluido, la anato- 


\section{TABLA 3.- TÉCNICAS PARA EXPONER LOS CANINOS MAXILARES INCLUIDOS}

\begin{tabular}{|l|l|}
\hline Técnica & Procedimiento \\
\hline Gingivectomía & $\begin{array}{l}\text { Encía queratinizada }>3 \mathrm{~mm} \text {. Se elimina la suficiente encía y se descubre entre la mitad y un } \\
\text { tercio de la corona del diente (7). }\end{array}$ \\
\hline reposición apical & $\begin{array}{l}\text { Incisión crestal de la zona edéntula para preservar la máxima cantidad de encía queratinizada. } \\
\text { Se realiza un colgajo de espesor total y en caso de encontrar hueso realizar una osteotomía con } \\
\text { cureta o fresa. Dos tercios de la corona deben quedar expuestos. Suturar el colgajo apicalmente } \\
\text { dejando sólo 2-3 mm de la corona cubierta (7). }\end{array}$ \\
\hline Técnica cerrada & $\begin{array}{l}\text { Colgajo usando una incisión crestal, se quita suficiente hueso alrededor del diente para poder } \\
\text { adherir el bracket. Se coloca un alambre o cadena que pasará por debajo del colgajo y a través } \\
\text { de la incisión. Antes de suturar, luxar suavemente con un periostotomo para asegurarnos que } \\
\text { no esté anquilosado (7). }\end{array}$ \\
\hline $\begin{array}{l}\text { Colgajo de espesor total, se localiza el diente incluido y se fenestra. El bracket se puede colocar } \\
\text { durante la cirugía o podemos poner un cemento quirúrgico para impedir que la herida } \\
\text { cicatrice (1). }\end{array}$ \\
\hline Tunelización & $\begin{array}{l}\text { Colgajo de espesor total, y se extrae el canino temporal. En este momento se evalúa el hueso } \\
\text { que recubre el diente incluido; osteotomía con cureta o fresa (4). } \\
\text { Se comprueba que existe una comunicación entre el diente incluido y el alveolo vacío del diente } \\
\text { temporal. Se adhiere un bracket al canino incluido y se pasa una cadena a través de túnel. Una } \\
\text { vez hecho todo esto, suturamos el colgajo en su posición inicial (7, 28). }\end{array}$ \\
\hline
\end{tabular}

mía de la zona edéntula, la posición vertical y horizontal inicial del canino; así como, la rapidez y dirección de la fuerza ortodóncica (8).

\section{2b. Técnicas para exponer el canino mandibular incluido}

Si hay suficiente espacio en la mandíbula que permita la ubicación del canino incluido en la arcada dental se debe considerar este tratamiento. Después de la exposición quirúrgica, en algunos casos el canino es capaz de erupcionar espontáneamente si tiene una angulación favorable. Si no se produce la erupción espontánea se debe combinar con la tracción ortodóncica. Según la posición escogeremos el acceso lingual o vestibular (12). En caso de conservar el canino temporal se puede escoger la tunelización para reposicionar el diente (30). El éxito de este tratamiento va a depender más de la edad del paciente que de la posición o el nivel de impactación del diente (27), si esto no fuera posible se deberían plantear otros tratamientos como la extracción o el transplante (2).

\section{Tratamiento ortodóncico final}

Durante esta fase, mientras el canino es alineado en la arcada dental se corrigen las rotaciones dentales que falten. Al final del tratamiento ortodóncico activo, se colocarán retenedores y el paciente se deberá citar periódicamente para control (4).

\section{Complicaciones en el tratamiento ortodóncico-quirúrgico}

La complicación más común es la falta de movimiento una vez iniciadas las fuerzas ortodóncicas, en estos casos se debería de pensar en realizar otro tipo de tratamiento sobre este canino. Esto pasa más 

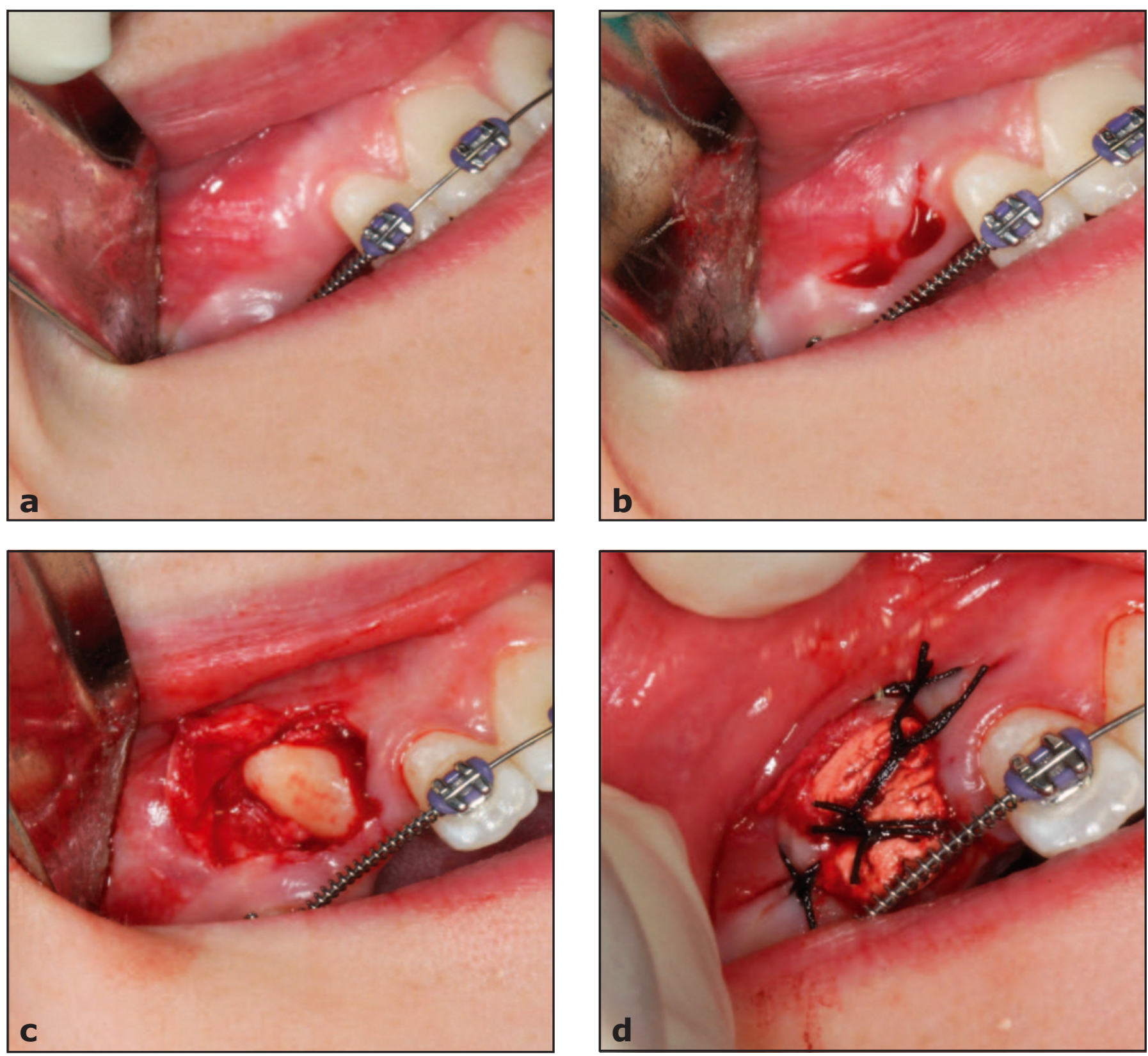

Fig. 2. Técnica abierta de exposición de los caninos incluidos. a) Vista antes de la fenestración; b) Colgajo de espesor total; c) Fenestración y exposición del diente; d) Cemento quirúrgico y sutura.

frecuentemente en los caninos incluidos en posición palatina. Las etiologías más comunes son:

1. No se ha quitado suficiente hueso alrededor de la corona del diente.

2. Mecanismo ortodóncico incorrecto.

3. Anquilosis. El diagnóstico de anquilosis se hace generalmente durante la cirugía, en estos casos se deben aplicar las fuerzas inmediatamente. En algunas ocasiones no es posible mover el diente y se ha de extraer.

4. Adhesión incorrecta (8).

Además de estas complicaciones habituales, Alessandri-Bonetti et al. (31) reportaron un caso de osteointegración de la cadena que traccionaba el canino provocando la no erupción de éste. Aunque 


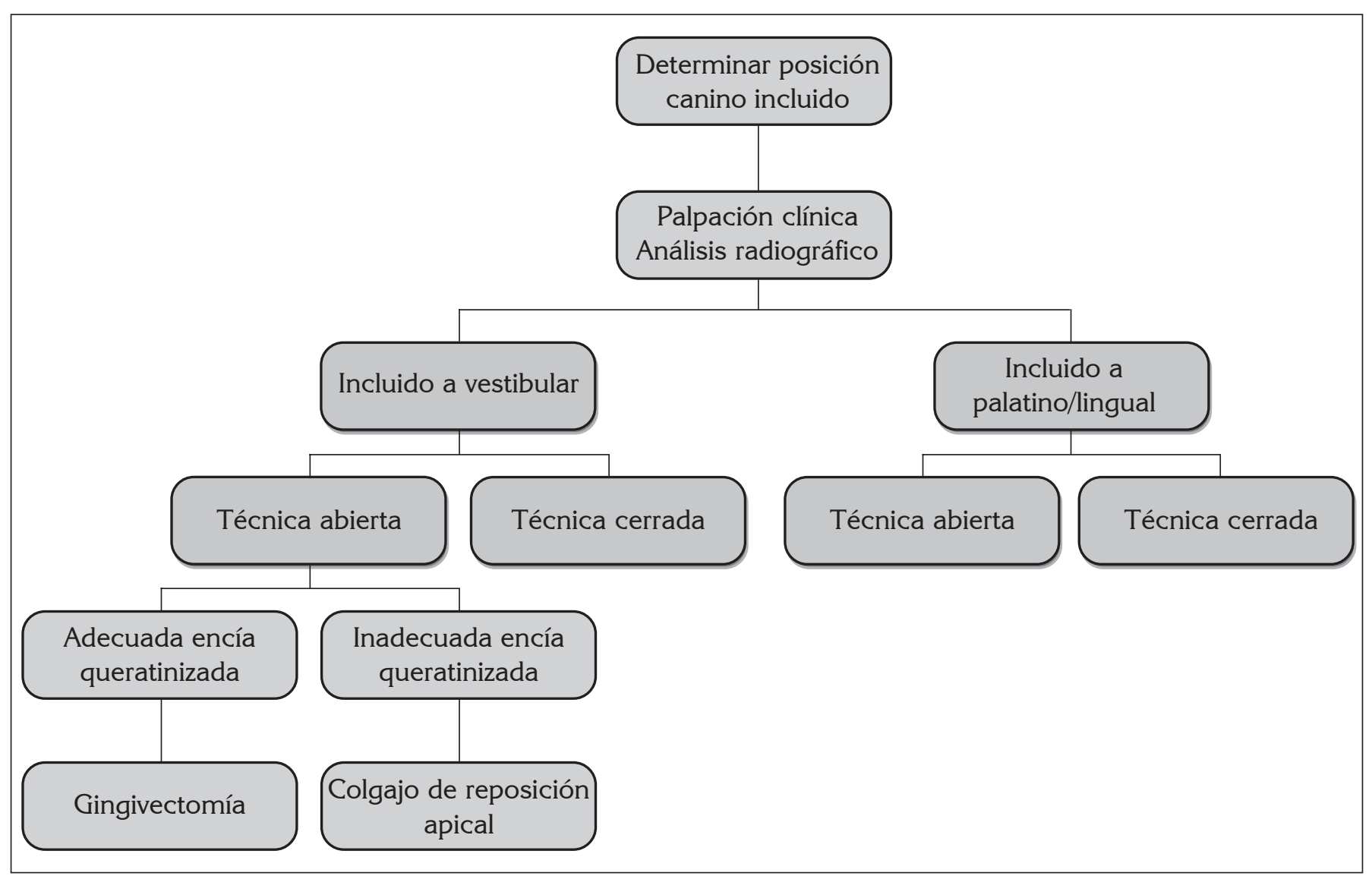

Fig. 3. Cuadro de decisión para escoger la técnica apropiada en un canino incluido, modificada de los criterios descritos por Cooke J, et al. (7).

se necesitarían más estudios que avalaran esta afirmación.

\section{Extracción}

La extracción (Figura 4) del canino incluido puede afectar a la estética y a la oclusión. Pero a veces es la única opción si hay limitaciones en llevar al canino a su posición en la arcada o su anatomía está afectada. En este caso se deberá decidir entre mover el premolar a la posición del canino u otras alternativas, como el autotrasplante o la restauración protética $(1,5,32,33)$.

\section{CONCLUSIONES}

Finalmente, después la revisión de la literatura actual, lo podemos resaltar tres puntos:
- Cuando tratamos caninos incluidos, es fundamental un apropiado diagnóstico para el éxito del tratamiento.

- El simple tratamiento interceptivo puede prevenir la inclusión del canino y hacer que erupciones correctamente en el arco dental. Siendo éste sin duda el mejor tratamiento posible.

- Las diferentes técnicas quirúrgicas y ortodóncicas nos permitirán corregir la inclusión y evitar las complicaciones de los caninos incluidos. Una selección cuidadosa de la técnica es esencial para el alineamiento exitoso de los caninos incluidos.

La bibliografía actual no aporta datos concluyentes sobre los métodos para exponer el canino incluido, por este motivo harían falta más estudios clínicos, ya que esta decisión queda a elección del equipo que efectúe el tratamiento. 


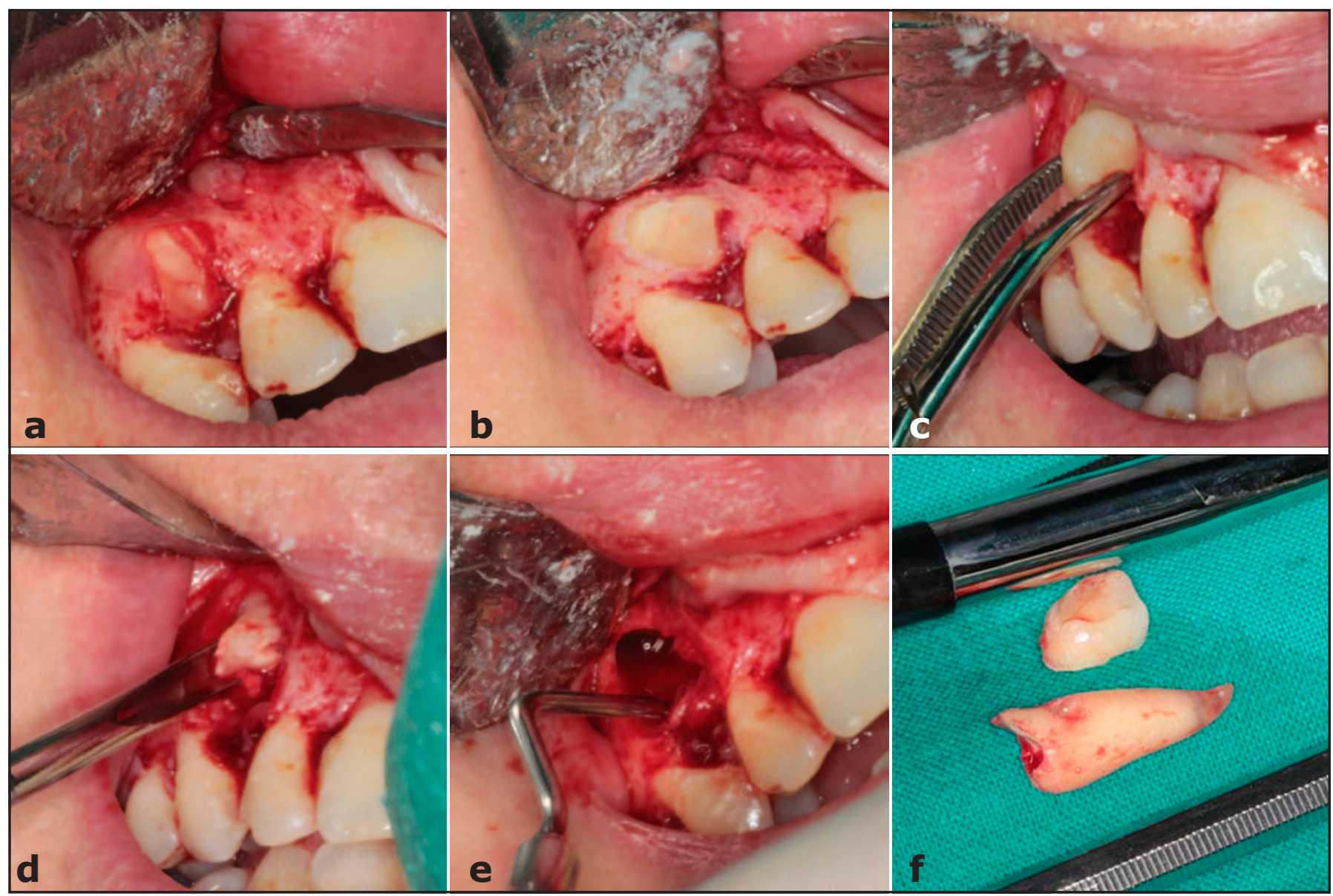

Fig. 4. Extracción de canino incluido. a) Colgajo de espesor total, b) Eliminación del hueso que recubre el diente; c) Odontosección de la corona; d) Luxación y avulsión de la raíz; e) Legrado de la cavidad; f) Visión de la corona y la raíz una vez extraídas.

\section{BIBLIOGRAFÍA}

1. Bedoya MM, Park JH. A review of the diagnosis and management of impacted maxillary canines. J Am Dent Assoc. 2009;140:1485-93.

2. Aras M, Halicioglu K, Yavuz M, Çağlaroğlu $M$. Evaluation of surgical-orthodontic treatments on impacted mandibular canines. Med Oral Patol Oral Cir Bucal. 2011;16:925-8.

3. Peng CL, Su YY, Lee SY. Unilateral horizontally impacted maxillary canine and first premolar treated with a double archwire technique. Angle Orthod. 2006;76:502-9.

4. Crescini A, Nieri M, Rotundo R, Baccetti T, et al. Combined surgical and orthdontic approach to reproduce the physiologic eruption pattern in impacted canines: report of 25 patients. Int $\mathrm{J}$ Periodontics Restorative Dent. 2007;27:529-37.

5. Sajnani AK, King NM. Retrospective audit of management techniques for treating impacted maxillary canines in children and adolescents over a 27-year period. J Oral Maxillofac Surg. 2011;69: 2494-9.

6. Litsas G, Acar A. A review of early displaced maxillary canines: etiology, diagnosis and interceptive treatment. Open Dent J. 2011;5:39-47.

7. Kolenc-Fusé FJ. Agenesias dentarias: en busca de las alteraciones genéticas responsables de la falta de desarrollo. Med Oral Patol Oral Cir Bucal. 2004; 9:385-95. 
8. Alqerban A, Jacobs R, Lambrechts P, Loozen G, et lal. Root resorption of the maxillary lateral incisor caused by impacted canine: a literature review. Clin Oral Investig. 2009;13:247-55.

9. Cooke J, Wang HL. Canine impactations: Incidence and management. Int $\mathrm{J}$ Periodontics Restorative Dent. 2006;26:483-91.

10. Chapokas AR, Almas K, Schincaglia GP. The impacted maxillary canine: a proposed classification for surgical exposure. Oral Surg Oral Med Oral Pathol Oral Radiol Endod. 2011;20:1-6.

11. Deepti A, Rayen R, Jeevarathan J, Muthu MS, et al. Management of an impacted and transposed maxillary canine. J Indian Soc Pedod Prev Dent. 2010; 28:38-41.

12. Nieri $M$, Crescini A, Rotundo R, Baccetti T, et al. Factors affecting the clinical approach to impacted maxillary canines: A Bayesian network analysis. Am J Orthod Dentofacial Orthop. 2010; 137:755-62.

13. Aras $M H$, Büyükkurt $M C$, Yolcu $U$, Ertaş $U$, et al. Transmigrant maxillary canines. Oral Surg Oral Med Oral Pathol Oral Radiol Endod. 2008;105: 48-52.

14. Celikoglu M, Kamak H, Oktay H. Investigation of transmigrated and impacted maxillary and mandibular canine teeth in a orthodontic patient population. J Oral Maxillofac Surg. 2010;68: 1001-6.

15. Parkin Nicola, Benson Philip E, Thind Bikram, Shah Anwar. Exposición quirúrgica abierta versus cerrada de dientes caninos desplazados hacia el techo de la boca (Revisión Cochrane traducida). En: La Biblioteca Cochrane Plus, 2008 Número 4. Oxford: Update Software Ltd. Disponible en: http://www.update-software.com. (Traducida de The Cochrane Library, 2008 Issue 3. Chichester, UK: John Wiley \& Sons, Ltd.). Accedido: 4 de Julio de 2012.

16. Pascual-Gil JV, Marqués-Mateo M, Puche-Torres M, Miragall-Alba L, et al. The meridian incisión: A technical modification in the conservative surgery of the impacted maxillary canine. Med Oral Patol Oral Cir Bucal. 2009;13:36-8.

17. Alessandri-Bonetti G, Zanarini $M$, Danesi $M$, Parenti SI, Gatto MR. Percentiles relative to maxillary permanent canine incination by age: a radiologic study. Am J Orthod Dentofacial Orthop. 2009;136:486-93.

18. Alessandri-Bonetti G, Incerti Parenti S, Zanarini M, Marini I. Double vs single primary teeth extraction approach as prevention of permanent maxillary canines ectopic eruption. Pediatr Dent. 2010;32:401-12.

19. Spencer HR, Ramsey R, Ponduri S, Brennan PA. Exposure of unerupted palatal canines: A survey of current practice in the United Kingdom, and experience of gingival-sparing procedure. $\mathrm{Br} \mathrm{J}$ Oral Maxillofac Surg 2010;48:641-4.

20. Zuccati G, Ghobadlu J, Nieri M, Clauser C. Factors associated with the duration of forced eruption of impacted maxillary canines: A restrospective study. Am J Orthod Dentofacial Orthop. 2006; 130:34956.

21. Naoumova J, Kurol J, Kjellberg H. A systematic review of the interceptive treatment of palatally displaced maxillary canines. Eur J Orthod. 2011; 33:143-9.

22. Milberg DJ. Labially impacted maxillary canines causing severe root resorption of maxillary central incisors. Angle Orthod 2006;76:173-6.

23. Becker A, Chaushu G, Chaushu S. Analysis of failure in the treatment of impacted maxillary canines. Am J Orthod Dentofacial Orthop. 2010; 137:743-54.

24. Scarfe WC, Farman AG, Sukovic P. Clinical applications of cone-beam computed tomography in dental practice. J Can Dent Assoc. 2006;72:75-80.

25. Pitt S, Hamdan A, Rock P. A treatment difficulty index of unerupted maxillary canines. Eur J Orthod. 2006;28:141-4. 
26. Zhang J, Zhang WJ, Wang XX, Xu JG, Huang Y. Orthodontic traction of impacted maxillary canine: a comparison of different ages. Shanghai Kou Qiang Yi Xue. 2006;15:130-2.

27. Yavuz MS, Aras $M H$, Büyükkurt MC, Tozoglu S. Impacted mandibular canines. J Contemp Dent Pract. 2007;8:78-85.

28. Bourzgui F, Belhaj S, Tazi H, Hamza M, Khazana MM. Surgical-orthodontic management of dental impaction. Int Orthod. 2009;7:257-67.

29. Schmidt AD, Kokich VG. Periodontal response to early uncovering, autonomous eruption, and orthodontic aligment of palatally impacted maxillary canines. Am J Orthod Dentofacial Orthop. 2007;131:449-55.

30. Patel S, Fanshawe T, Bister D, Cobourne MT. Survival and success of maxillary canine autotransplantation: a retrospective investigation. Eur J Orthod. 2011;33:298-304.

31. Crescini A, Baccetti T, Rotundo R, Mancini EA, Prato GP. Tunnel technique for the treatment of impacted mandibular canines. Int J Periodontics Restorative Dent. 2009; 29:213-8.

32. Alessandri-Bonetti G, Incerti Parenti S, Daprile G, Montevecchi M. Failure after closed traction of an unerupted maxillary permanent canine: Diagnosis and treatment planning. Am J Orthod Dentofacial Orthop. 2011;140:121-5.

33. Calvo-Guirado J, Ortiz-Ruiz AJ, López-Marí L, Delgado-Ruiz R, Maté-Sánchez J, Bravo González LA. Inmediate maxillary restoration of single tooth implants using platform switching for crestal bone preservation: A 12-month study. Int J Oral Maxillofac Implants. 2009;24:275-81.

\section{CORRESPONDENCIA}

José López López

Campus Universitario de Bellvitge

Pabellón de Gobierno, 2 planta, despacho 2:29

Feixa Llarga, s/n

08907 L'Hospitalet de Llobregat

Correo electrónico: 18575jll@gmail.com 